\title{
Atuação do enfermeiro frente aos efeitos da circulação extracorpórea
}

\author{
Camila Sartorio*, Daniele Cristina Rocha Lima*, Flaviana de Castro Metello*, \\ Olga Carla Ribeiro Costa Soares*, Valéria Cristina Moreira de Medeiros Mota*, Flávia Silva de Souza, M.Sc.**
}

* Enfermeira, aluna do Curso de Extensão para graduandos e enfermeiros no Hospital Universitário Clementino Fraga Filho, HUCFF, **Professora Orientadora, Chefe da Seção de Educação Continuada do Serviço de Desenvolvimento em Enfermagem do Hospital Universitário Clementino Fraga Filho (UFRJ), docente da disciplina de UTI da UNIPLI

\section{Resumo}

A circulação extracorpórea (CEC) é uma técnica mundialmente utilizada nos casos em que o coração precisa parar de bater para que ocorra a cirurgia. A máquina faz o papel do coração de bombear sangue de volta para o corpo e do pulmáo, oxigenando o sangue venoso que chega dos tecidos. Assim este estudo teve por objetivos: identificar as principais complicaçóes ocasionadas pela CEC e discutir a conduta do enfermeiro na minimizaçáo dos efeitos indesejáveis causados pela CEC. Trata-se de uma revisão da literatura, de natureza descritiva e exploratória. A coleta de dados foi realizada através da base de dados da Biblioteca Virtual de Saúde (BVS), no recorte temporal de 10 anos, sendo utilizados os seguintes descritores: cirurgia torácica, CEC e complicações pós-operatórias. Foram encontrados 8 trabalhos dos quais também surgiram as unidades temáticas: Complicações pulmonares permanecendo como grande problema no pós-operatório em cirurgias com CEC e Atuação do enfermeiro frente aos efeitos da CEC. Conclui-se que as cirurgias que utilizam a CEC podem levar a complicaçóes que, se não forem evitadas ou minimizadas, poderão desenvolver alteraçôes severas e de difícil reversão. Das complicaçôes evidenciadas a mais comum está relacionada à mecânica ventilatória.

Palavras-chave: cirurgia torácica, circulação extracorpórea, complicaçôes pós-operatórias.

\section{Abstract}

\section{Nurse's role in the management of cardiopulmonary bypass effects}

Extracorporeal circulation (ECC) is a technique used worldwide in cases in which the heart must stop beating for a short time during surgery. The machine takes over the function of the heart to pump blood around the body and lungs, oxygenating venous blood coming from tissues. This is a literature review with descriptive and exploratory approach which aimed at identifying the major complications caused by ECC and discussing the importance of nurses performance in minimizing the adverse effects caused by ECC. Data collection was performed through Virtual Health Library (VHL) database, using articles published during the last 10 years. Eight articles were found and two categories emerged: Pulmonary complications still as 
a big problem after surgery with ECC and Nurse's role in the management of cardiopulmonary bypass effects. We conclude that surgery using ECC can lead to complications which, if not avoided or minimized, may develop significant status changes and difficult to reverse. It was also evidenced that the more common complications are related to ventilatory mechanics.

Key-words: thoracic surgery, extracorporeal circulation, postoperative complications.

\section{Resumen}

\section{La actuación del enfermero ante los efectos de la circulación extracorpórea}

La circulación extracorpórea (CEC) es una técnica mundialmente utilizada en casos de que sea necesario que el corazón deje de latir para realizar la cirugía. La máquina asume las funciones del corazón encargándose de la acción de bombeo para enviar sangre hacia los pulmones y el resto del cuerpo, oxigenando la sangre venosa que llega a los tejidos. Esta revisión de literatura de naturaleza descriptiva y exploratoria tiene como objetivos: identificar las principales complicaciones ocasionadas por la CEC y discutir la importancia de la actuación del enfermero en la minimización de los efectos no deseados causados por la CEC. Los datos fueron colectados a través de la base de datos de la Biblioteca Virtual en Salud (BVS), utilizando estudios publicados en los últimos 10 ańos. Fueron encontrados 8 estudios y surgieron dos unidades temáticas: Complicaciones pulmonares permaneciendo como gran problema en el post-operatorio en cirugías con CEC y Actuación del enfermero frente a los efectos de la CEC. Se concluye que las cirugías que utilizan la CEC pueden llevar a complicaciones que, si no se pueden evitar o minimizar, podrán desarrollar alteraciones severas y de difícil reversión. En relación a las complicaciones evidenciadas, la más común está asociada a la mecánica ventilatoria.

Palabras-clave: cirugía torácica, circulación extracorpórea, complicaciones postoperatoria, atención de enfermería.

\section{Introdução}

A Circulação Extracorpórea (CEC), em um sentido mais amplo, compreende o conjunto de máquinas, aparelhos, circuitos e técnicas, mediante as quais, substituem temporariamente as funçóes do coração e dos pulmóes enquanto esses órgãos ficam excluídos da circulação.

É quase impossível reparar defeitos cardíacos por meios cirúrgicos enquanto o coração ainda estiver bombeando. Por conseguinte, muitos tipos de máquinas coração-pulmão artificiais foram desenvolvidas para assumir o lugar do coração e dos pulmóes durante o curso dessas cirurgias. Tal sistema é referido como circulação extracorpórea. $\mathrm{O}$ sistema consiste, principalmente, em uma bomba e um aparelho de oxigenaçáo, onde quase todos os tipos de bomba, que não produzem hemólise do sangue, parecem apropriadas [1].

Os métodos usados para oxigenar o sangue incluem: 1) borbulhar oxigênio pelo sangue e remover as bolhas do sangue antes de introduzi-lo novamente no paciente; 2) fazer o sangue gotejar sobre superfícies de folhas plásticas, na presença de oxigênio; 3) passar o sangue sobre as superfícies de discos giratórios e; 4) passar o sangue entre membranas delgadas ou por tubos delgados que sejam permeáveis ao oxigênio e ao dióxido de carbono [2].
Os diferentes sistemas apresentam variadas dificuldades, incluindo a hemólise do sangue, o desenvolvimento de pequenos coágulos no sangue, a probabilidade de pequenas bolhas de oxigênio ou pequenos êmbolos de agente antiespumante passando para as artérias do paciente, a necessidade de grande quantidade de sangue para preparar o sistema, a falha em trocar quantidades adequadas de oxigênio e a necessidade de utilizar heparina para impedir a coagulação do sangue no sistema extracorpóreo. A heparina também interfere com a hemostasia adequada durante o procedimento cirúrgico. Todavia, a despeito dessas dificuldades, nas mãos de especialistas os pacientes podem ser mantidos vivos em máquinas coração-pulmão artificiais por muitas horas, enquanto as operaçóes são realizadas no interior do coração [2].

Em virtude de sua complexidade, da multiplicidade de componentes mecânicos e de suas interaçôes com o sangue, a CEC é capaz de produzir uma grande variedade de alteraçóes no organismo humano, que de modo geral, surgem imediatamente após a operação ou na unidade de terapia intensiva [1].

O bombeamento artificial do sangue e a sua circulação por superfícies plásticas rígidas ou pouco flexíveis e não biológicas produzem traumatismo e injuria aos elementos celulares e proteicos, diretamente proporcional ao tempo de duração do 
procedimento. Embora este traumatismo seja bem tolerado pela maioria dos pacientes, em determinadas circunstâncias os seus efeitos adversos são mais pronunciados e podem tornar-se clinicamente aparentes, contribuindo substancialmente para o desenvolvimento de complicaçóes após a operação [1].

Uma das características dos pacientes submetidos à cirurgia cardíaca com circulação extracorpórea é a facilidade com que os demais sistemas orgânicos podem ser afetados, por alteraçóes primárias da função do sistema cardiovascular. As funçóes pulmonares, neurológicas, renais e hepáticas, principalmente, dependem da adequada e contínua nutrição e, rapidamente, colapsam diante de reduçóes do débito cardíaco [1].

A literatura mostra que as principais complicaçóes que ocorrem nos pacientes submetidos à CEC são: as hemorragias, o baixo débito cardíaco, as arritmias cardíacas, a insuficiência respiratória, a insuficiência renal, as alteraçóes neurológicas ou neuropsiquiátricas, as alteraçóes hidroeletrolíticas, as alterações abdominais e outras mais raramente observadas [2].

A interação do enfermeiro se faz necessária para a identificação de complicaçóes já instaladas ou de pequenos desvios que, se não corrigidos, poderão desenvolver alterações severas e de difícil reversão. É necessário salientar que tal intervenção do enfermeiro acontece, entre outros momentos, durante a realização da CEC, sobretudo, pois consideramos que muitos enfermeiros são responsáveis pela dinâmica da perfusão.

Contudo, observamos também que, por vezes, o enfermeiro que atua na Unidade de Terapia Intensiva Pós-operatória (UTI PO) realiza vigilância contínua desses pacientes, assim como intervençóes necessárias, quando é solicitado ou quando prescritas pela equipe médica. É fato, de que no momento da recepçáo do paciente em UTI PO, o enfermeiro nem sempre recebe as informaçóes pertinentes ao transcorrer daquela cirurgia, sejam estas passadas pelo médico ou pelo anestesista, ou pelo profissional responsável pela CEC.

No momento recepção deste paciente ao leito, observa-se uma preocupação com o aquecimento corporal, a estabilidade hemodinâmica, a ventilaçáo mecânica, a drenagem de secreçôes, as questôes administrativas que envolvem a internação do paciente na unidade, porém o enfermeiro nem sempre está presente durante a troca de informaçóes do transcorrer da cirurgia, o que denota que ele desco- nhece sobre as intercorrências em sala de operação, assim como, a história clínica do paciente antes do procedimento. Portanto, as intervençóes realizadas tentam suprir as necessidades evidenciadas naquele momento e não há tempo hábil para que o enfermeiro e sua equipe se preparem antes de receber o paciente na UTI PO.

Existem instituições que trabalham com protocolos nos quais, antes de transferir o paciente do Centro Cirúrgico, a enfermeira do setor e o perfusionista informam ao enfermeiro da UTI PO tudo o que aconteceu durante o procedimento, assim, este último se organiza com sua equipe para receber o paciente em quaisquer condiçóes clínicas, hemodinâmicas e cirúrgicas que ele estiver. Entretanto a dinâmica do setor, as condiçóes de trabalho, por vezes, não permitem que o enfermeiro possa participar deste processo, táo importante para a garantia de um período pós-operatório com o mínimo de complicaçóes possíveis.

A motivaçáo em realizar este estudo surgiu a partir de vivência em treinamento em serviço do curso de extensão no CTI Cardíaco de um Hospital Universitário localizado na cidade do Rio de Janeiro. Presenciou-se o pós-operatório de diversas cirurgias cardíacas e observamos algumas complicaçóes causadas pela CEC e a falta da troca de informaçóes entre as equipes sobre o transcorrer da cirurgia, o tempo de CEC e qual o quadro real daqueles pacientes no momento que seriam transferidos para a UTI PO, o que nos levou a investigar a pesquisa em questáo.

Entende-se como problema de pesquisa: De que forma o enfermeiro que trabalha na UTI PO pode atuar frente às principais complicaçóes causadas pela CEC no pós-operatório imediato de cirurgia cardíaca?

Assim, tem-se como objeto de estudo a atuação do enfermeiro frente às principais complicaçóes causadas pela CEC no pós-operatório imediato de cirurgia cardíaca.

Este estudo se justifica pelo fato de que a utilização da CEC, durante as cirurgias, pode estar associada a várias mudanças na fisiologia normal do organismo. As múltiplas causas destas alteraçóes interagem e representam um número de rotas potenciais para a disfunção orgânica pós-operatória.

Torna-se essencial a abordagem deste tema, uma vez que, na práxis diária ao paciente pós-operatório de cirurgia cardíaca, podemos nos deparar com situaçóes e complicaçóes advindas dos efeitos adversos em consequência da CEC, 
principalmente em decorrência do tempo exposto ao procedimento. $\mathrm{O}$ profissional enfermeiro deve conhecer a técnica, acompanhar a evolução do paciente, estando atento à situação hemodinâmica, e preparado para intervir nas diversas situaçóes que podem ser encontradas.

Para responder o problema de estudo, estabeleceram-se os seguintes objetivos: 1) Identificar as principais complicaçóes ocasionadas pela CEC através de revisão bibliográfica; 2) Discutir a conduta do enfermeiro na minimizaçáo dos efeitos causados pela CEC.

\section{Material e métodos}

Trata-se de um estudo descritivo e exploratório. O estudo exploratório tem como objetivo tornar mais explícito o problema, aprofundar as ideias sobre o objeto do estudo [3]. Enquanto o estudo descritivo é a simples descrição de um fenômeno, o exploratório permite ao investigador aumentar sua experiência de determinado problema [4].

Trata-se de uma revisão de literatura, pois é desenvolvida a partir de material já elaborado, constituído principalmente de artigos científicos e livros [5].

O método da revisão integrativa possibilita sumarizar as pesquisas já concluídas e obter conclusóes a partir de um tema de interesse [5].

Os dados foram coletados através de pesquisa nas bases de dados virtuais da Biblioteca Virtual de Saúde (BVS), com consulta através da apresentação dos descritores: Cirurgia Torácica, CEC e Complicaçóes Pós Operatórias.

Inicialmente, procurou-se pelos descritores de forma individual, o que proporcionou um vasto acervo de trabalhos científicos, conforme Figura I.

Figura I - Apresentação quantitativa dos descritores de forma individual.

\begin{tabular}{|l|c|c|c|c|}
\hline Descritores & Lilacs & IBECS & Medline & Scielo \\
\hline Cirurgia Torácica & 1.442 & 100 & 8.312 & 588 \\
\hline CEC & 673 & 63 & 13.557 & 335 \\
\hline $\begin{array}{l}\text { Complicações Pós } \\
\text { Operatórias }\end{array}$ & 4.156 & 1.366 & 242.343 & 314 \\
\hline TOTAL & 6.041 & 1.529 & 264.212 & 1.237 \\
\hline
\end{tabular}

Observou-se que este vasto conjunto de trabalhos necessitava de um refinamento para que houvesse a possibilidade de ser realizada uma leitura seletiva dos artigos. Desta forma, retornou-se à BVS, desta vez, com os descritores e dupla e trio, para que se conseguisse alcançar o acervo específico.

Assim, apresenta-se a seguir os descritores em dupla e trio e o quantitativo de trabalhos encontrados, conforme Figura II.

Figura II - Apresentação quantitativa dos descritores em dupla e trio.

\begin{tabular}{|l|c|c|c|c|}
\hline \multicolumn{1}{|c|}{ Descritores } & Lilacs & IBECS & Medline & Scielo \\
\hline $\begin{array}{l}\text { Cirurgia Torácica } \\
+ \text { CEC }\end{array}$ & 150 & 4 & 58 & 12 \\
\hline $\begin{array}{l}\text { CEC + Complica- } \\
\text { ções Pós Opera- } \\
\text { tórias }\end{array}$ & 65 & 21 & 1.787 & 12 \\
\hline $\begin{array}{l}\text { Cirurgia Torácica } \\
+ \text { CEC + Com- } \\
\text { plicações Pós } \\
\text { Operatórias }\end{array}$ & 15 & 1 & 11 & 0 \\
\hline TOTAL & 230 & 26 & 1.856 & 24 \\
\hline
\end{tabular}

Após esta fase, iniciou-se a leitura interpretativa dos textos, que significa entender a intenção do autor, julgar o material coletado em relação ao critério de verdade e cuidar para que todas as informaçóes sejam comprovadas [6] para posteriormente, submetê-los à Análise Temática, apreensão do conteúdo, isto é, tema, problemas, ideias (central e secundárias), raciocínio e argumentação [6].

Para ser realizada esta Análise Temática dos artigos encontrados, um total de sete, utilizou-se como critérios de inclusão: não serem repetidos, acessibilidade por meio virtual, trabalhos em língua portuguesa, trabalhos dos últimos 10 anos e responderem aos objetivos de pesquisa.

Dentre os trabalhos consultados, observou-se que todos eram do tipo ensaios clínicos, pesquisas baseadas em evidências, realizados por médicos e fisioterapeutas. Não foram encontrados trabalhos escritos por enfermeiros, o que remete a necessidade de ampliar os horizontes e de se escrever sobre a prática assistencial, também baseada em evidência para se cuidar melhor.

\section{Resultados e discussão}

Após a coleta de dados, os mesmos foram separados, codificados e categorizados, para depois sofrerem a abordagem qualitativa. Utilizou-se a análise temática como técnica de distribuição e compreensáo desses dados. A análise temática pro- 
porcionou responder os objetivos e identificar duas unidades temáticas que serão apresentadas a seguir.

\section{Complicaçóes pulmonares permanecendo como grande problema no pós-operatório em cirurgias com CEC}

Esta primeira categoria refere-se ao levantamento dos dados sobre as principais complicaçóes decorrentes da CEC no pós-operatório imediato de cirurgia cardíaca. Dentre os seis artigos encontrados, quatro citam a complicação pulmonar como a maior incidência, seguida das arritmias cardíacas e por último a Insuficiência Renal aguda, com necessidade de diálise:

- 34\% dos pacientes apresentaram atelectasia $[7,12]$

- $31 \%$ apresentaram derrame pleural, relacionados ao maior tempo de CEC $[7,12]$;

- Em 17 e 33\% a fibrilação atrial e flütter foram observados nos primeiros dias após RVM [8];

- Entre 12 e 31,6\% de cirurgias para correção de aneurismas com CEC apresentaram complicaçóes respiratórias e mortalidade de 16,7\% [9];

- $16,1 \%$ apresentaram insuficiência renal aguda, sendo que 4,9\% necessitaram de diálise como terapia de substituição renal [10].

Um estudo sobre os custos da RVM sem CEC evidenciou outras complicaçóes observadas: AVE, IAM, infecçáo respiratória e de feridas [11].

Em virtude das complicaçóes que acometem o paciente no pós-operatório da cirurgia cardíaca com $\mathrm{CEC}$, a função pulmonar encontra-se em destaque com aumento de morbidade e mortalidade causadas pela perfusão pulmonar diminuída em sua capacidade residual funcional. É necessária uma assistência minuciosa e adequada do enfermeiro para que haja melhor identificação nas alteraçóes dos parâmetros ventilatórios com o intuito de minimizar maiores agravos.

As complicaçóes pulmonares são causas importantes de morbidade e mortalidade nos pacientes submetidos à cirurgia cardíaca com CEC. $\mathrm{Na}$ circulação pulmonar há aumento da água extravascular com preenchimento alveolar por células inflamatórias que levam à inativação do surfactante pulmonar e colabamento de algumas áreas, com modificação na relação ventilação/perfusão pulmonar, diminuição de sua complacência e aumento do trabalho respiratório no período pós-operatório [7:2]. O grau de alteraçáo funcional pulmonar depende de vários fatores, como a função pulmonar pré-operatória, o tipo de cirurgia, o tempo de circulação extracorpórea, a intensidade da manipulação cirúrgica, o número de drenos pleurais colocados e o tempo de cirurgia [7:3]. Uma assistência ventilatória adequada utilizada durante o período perioperatório pode minimizar as alterações da função pulmonar, fazendo com que haja uma diminuição nas complicaçôes pós-operatórias [7].

As alterações da função pulmonar em pacientes submetidos à cirurgia cardíaca com circulação extracorpórea são em grande parte responsáveis pela morbidade desses pacientes [7:6]. As atelectasias são as complicaçóes mais frequentes [7:7], ocasionadas por diminuição da capacidade residual funcional [7:8], pelas alteraçóes da mecânica da caixa torácica e do tecido pulmonar [13:9], por aumento da resistência das vias aéreas [7:10] , pela dor pós-operatória [7:11] e por paralisia do nervo frênico [13:12,14], dentre outros fatores. Adicionalmente, a circulação extracorpórea ocasiona resposta inflamatória sistêmica que pode contribuir para a diminuição da $\mathrm{PaO} 2 / \mathrm{FiO} 2$ [7:14,15].

Uma característica importante desta categoria é que, diferente do que encontramos na literatura vigente - a inflamação como a principal complicação - os ensaios clínicos evidenciaram que a atelectasia devido à reduçáo da complacência e da própria ventilação durante a CEC é a complicação mais comum, o que nos faz refletir sobre as pesquisas baseadas em evidências clínicas como guia prático de diagnóstico e tratamento.

Outro ponto importante é que, diferente do observado na prática, as alteraçóes pulmonares foram mais evidentes do que as alteraçóes renais. Isto pode estar relacionado também com o tipo de clientela atendida no hospital universitário. Uma característica marcante é que hospitais universitários, em sua maioria, são referenciados e tratam de pessoas com diversos níveis de complexidade. Portanto, provavelmente a inflamação e as disfunçóes contráteis cardíacas podem ser consideradas alteraçóes clínicas dos pacientes que procuram atendimento nesse hospital.

Reforça-se a necessidade de reflexão de que a enfermagem também pode atuar baseada em evidências clínicas, do processo de enfermagem, que se constituem de dados confiáveis para conduzir sua prática de forma integrativa e com qualidade. 


\section{Atuaçáo do enfermeiro frente aos efeitos da cir- culação extracorpórea}

Esta segunda categoria refere-se à atuação do enfermeiro frente às complicaçóes decorrentes da CEC, principalmente a mais comum que é a respiratória, evidenciada pela pesquisa bibliográfica.

Portanto, o enfermeiro tem papel fundamental na recuperação da saúde dos pacientes submetidos à cirurgia com utilização da circulação extracorpórea. No momento da admissão do paciente na UTI, o enfermeiro deve verificar com o cirurgião e com o anestesista vários itens de fundamental importância para um adequado pós-operatório, como: diagnóstico inicial, procedimento realizado e tempo de circulação extracorpórea [13].

A transferência do paciente para a UTI deverá ser rápida e segura, sendo realizada em conjunto pelas equipes da cirurgia e de anestesia, bem como a equipe de enfermagem, tendo o enfermeiro na liderança da açáo procedendo a monitorização de toda hemodinâmica do paciente, atenção para não haver perdas de drenos, cateteres e sondas, hipoventilação, deslocamento de tubo endotraqueal ou de linhas venosas e alteração do tempo de infusão de drogas e/ou hipotermia [7].

O enfermeiro deve utilizar de condutas iniciais como: posicionar o paciente adequadamente no leito, identificar acessos vasculares, abertura de drenos torácicos, verificar sondas, parâmetros do respirador, fazer avaliação física, exame de rotina e medicaçôes prescritas.

Uma adequada assistência da equipe de profissionais de enfermagem deve ser capaz de evitar ou minimizar possíveis complicaçóes em pacientes potencialmente mais graves, visando também à redução do tempo da UTI [1].

Torna-se necessário conhecer o perfil dos pacientes, bem como as complicaçóes mais incidentes provocadas pela circulação extracorpórea na realidade da instituição em que se trabalha, com objetivo de levantar propostas para a qualificação da equipe de enfermagem frente às demandas do cuidado [13].

Os enfermeiros devem conhecer e estar preparados para identificar e tratar as complicaçóes em potencial nesses pacientes. As principais complicaçôes que podem ocorrer no pós-operatório de cirurgia com utilização da circulação extracorpórea são: alteração do débito cardíaco, alteração da contratilidade da pré-carga e pós-carga, hipertensão arterial sistêmica, hipertensão pulmonar, alteraçóes da frequência e ritmos cardíacos, pneumotórax, atelectasia, insuficiência renal, distúrbios hemorrágicos, entre outros [14].

De acordo com tantas complicaçóes, conclui-se que se faz necessário ter conhecimento prévio das necessidades de enfermagem e complicaçóes em pacientes submetidos à cirurgia com circulação extracorpórea, para proporcionar-lhes uma assistência de excelência e qualidade [14].

Quanto maior o tempo de circulação extracorpórea, tanto maiores serão as chances de ocorrer complicações.

No caso da complicação respiratória, torna-se necessária uma assistência qualificada do enfermeiro com a realização de ausculta pulmonar, análise de RX diariamente, avaliar os parâmetros ventilatórios e executar juntamente com o fisioterapeuta um trabalho de recrutamento alveolar com a finalidade de evitar colapsos alveolares, assim como desfazer e mobilizar secreçóes brônquicas. Tudo isto, diante de percepçáo, conhecimento científico, e pensamento crítico, proporcionando qualidade na assistência.

O enfermeiro deve seguir uma conduta clínica que é entendida como um conjunto articulado de saberes e de açóes com intuito de atender a necessidade apresentada pelo cliente e identificada pelo enfermeiro durante a assistência, norteada pelo pensamento crítico - o processo de enfermagem [7].

$\mathrm{O}$ enfermeiro, por ser um profissional com maior grau de proximidade com o paciente e familiares, está mais capacitado para avaliar o processo educativo, levantando as necessidades educacionais destes indivíduos, suas crenças e valores, o autoconhecimento de suas condiçóes de saúde e serviços de apoio existentes para o processo de reabilitação dos pacientes [15].

\section{Conclusão}

Com a elaboração deste trabalho, constatou-se que a CEC é um procedimento cirúrgico cuja descoberta e aperfeiçoamento, ao longo dos tempos, revelaram-se fundamentais para a realização de cirurgias cardíacas e cirurgias em que há a necessidade de substituir funcionalmente o coração, permitindo assim manter o organismo vivo no período intraoperatório.

É importante ressaltar que o enfermeiro é figura essencial no cuidado aos indivíduos com indicação cirúrgica, independente do período operatório em que se encontram. Particularmente 
no que concerne à CRM, ele deve participar do cuidado do paciente desde o momento da decisão de realização da cirurgia, até, muitas vezes, o pós-operatório tardio.

Dentre as complicaçóes relacionadas à CEC, a mais comum evidenciada foi a respiratória, que pode estar relacionada à diminuição da complacência pulmonar. Assim o paciente que permanece mais tempo em CEC pode apresentar no pós-operatório: atelectasia, infecção pulmonar e SDRA, tornando-a uma complicação grave. Desta forma, o enfermeiro precisa ter conhecimento profundo sobre ventilação mecânica para proporcionar um cuidado mais individualizado e eficaz.

Espera-se que este estudo abra caminhos para outras pesquisas, tentando melhor capacitaçáo do enfermeiro frente às complicaçóes causadas pela circulação extracorpórea, despertando não apenas o interesse, mas o entusiasmo, ocasionando assim o aumento das publicaçóes científicas pelos enfermeiros que tanto contribuem para o conhecimento e principalmente a melhora da qualidade da assistência de enfermagem.

\section{Referências}

1. Souza MHL, Elias DO. Fundamentos da circulação extracorpórea. 2a ed. Rio de Janeiro: Centro Alfa; 2006. 809 p.

2. Guyton AC, Hal JE. Tratado de Fisiologia Médica. 11 ${ }^{\mathrm{a}}$ ed. Rio de Janeiro: Saunders Elsevier; 2006. 276 p.

3. Alves M. Como escrever teses e monografias: um roteiro passo a passo. Rio de Janeiro: Campus; 2003.

4. Triviños ANS. Introduçáo à pesquisa em ciências sociais: a pesquisa qualitativa em educaçáo. São Paulo: Atlantas; 1990.

5. Matsubara TC, Carvalho EC, Canini SRMS, Sawada NO. A crise familiar no contexto do Transplante de
Medula Óssea (TMO): uma revisão integrativa. Rev Latinoam Enfermagem 2007;15(4):665-70.

6. Marconi MA, Lakatos EM. Metodologia do trabalho científico. 7a ed. São Paulo: Atlas; 2007. 225 p.

7. Barbosa RAG, Carmona MJC. Avaliaçáo da funçáo pulmonar em pacientes submetidos à cirurgia cardíaca com circulação extracorpórea. Rev Bras Anestesiol 2002;52:6:689-99.

8. Lúcio EA, Flores A, Blacher C, Leães PE, Lucchese FA, Ribeiro JP. Efetividade do metoprolol na prevenção de fibrilaçáo e flutter atrial no pós-operatório de cirurgia de revascularizaçáo miocárdica. Arq Bras Cardiol 2003;82(1):37-41.

9. Rojas SSO, Veiga VC, Souza JM, Berlinck MF, Senra DF, Vieira RW et al. Correçáo dos aneurismas da aorta torácica e toracoabdominal. Técnica de Canulação Central. Arq Bras Cardiol 2005;84(4):297-302.

10. Santos FO, Silveira MA, Maia RB, Monteiro MDC, Martinelli R. Insuficiência renal aguda após cirurgia de revascularização miocárdica com circulação extracorpórea - incidência, fatores de risco e mortalidade. Arq Bras Cardiol 2004;83(2):145-8.

11. Girardi PBMA, Hueb W, Nogueira CRSR, Takiuti ME, Nakano T, Garzillo CL et al. Custos comparativos entre a revascularização miocárdica com e sem circulação extracorpórea. Arq Bras Cardiol 2008;91(6):369-76.

12. Alcântara EC, Santos VN. Estudo das complicaçôes pulmonares e do suporte ventilatório náo invasivo no pós-operatório de cirurgia cardíaca. Rev Med Minas Gerais 2009;19(1):5-12.

13. Fernandes MVB, Aliti G, Souza EN. Perfil de pacientes submetidos à cirurgia de revascularização miocárdica: Implicaçôes para o cuidado de enfermagem. Rev Eletrônica Enferm 2009;11(4):993-9.

14. Rocha LA, Maia TF, Silva LF. Diagnóstico de enfermagem em pacientes submetidos à cirurgia cardíaca. Rev Bras Enferm 2006;59(3)321-6.

15. Dantas RAS, Aguillar OM. Problemas na recuperação de pacientes submetidos à cirurgia de revascularização do miocárdio: $\mathrm{O}$ acompanhamento pelo enfermeiro durante o primeiro mês após a alta hospitalar. Rev Latinoam Enferm 2001;9(6):31-6. 